\title{
Do Radiologists Report the TNM Staging in Radiology Reports for Head and Neck Cancers? A National Survey Study
}

\author{
(D). Ko, (D). Parvathaneni, (DP.A. Hudgins, and DY. Anzai
}

\begin{abstract}
BACKGROUND AND PURPOSE: CT and MR imaging are widely used for the staging of head and neck cancer. Currently, there are no data regarding whether the primary tumor, nodes, metastasis (TNM) staging is routinely incorporated into radiology reports. We conducted a national survey to determine whether radiologists routinely address staging, in particular regarding $\mathrm{T}$ (primary tumor) and $\mathrm{N}$ (nodal).
\end{abstract}

MATERIALS AND METHODS: The survey was sent to 782 members of the American Society of Head and Neck Radiology. The survey asked whether they assign TN staging in reports. If they do assign TN staging, what are the reasons for doing so, and if not, what are the barriers or reasons for not including it in the radiology report? The method of measuring the size of the primary tumor and pathologic lymph nodes was also queried.

RESULTS: A total of 229 responses were returned (29.3\% response rate). Approximately half (49\%; $95 \%$ confidence interval, $43.55-54.5 \%)$ of the responders thought that incorporating TN staging is important. However, only $24.5 \%$ (95\% confidence interval, $19.8 \%-29.2 \%$ ) stated that they routinely assigned TN staging in their radiology reports. The most common barriers were being afraid of being inaccurate (59\%) and being unable to remember the staging classifications (58.2\%); $76.9 \%$ indicated that they measure a primary tumor in 3D.

CONCLUSIONS: Staging head and neck cancer based on imaging presents unique challenges. Nearly half of the responding radiologists think it is important to incorporate TN staging in radiology reports, though only a quarter of them routinely do so in practice.

ABBREVIATIONS: H\&N = head and neck; TNM = (primary) tumor, nodes, metastasis

T and MR imaging are widely used for the staging of a newly

- diagnosed head and neck $(\mathrm{H} \& \mathrm{~N})$ squamous cell carcinoma. ${ }^{1-4}$ Treatment decisions depend on various factors, including the stage of disease, pathologic type, comorbidities, certain risk factors such as human papillomavirus status, and the patient's preference regarding treatment. Consistent, accurate, and precise characterization of $\mathrm{H} \& \mathrm{~N}$ cancer on the imaging report is thus an integral component of staging of $\mathrm{H} \& \mathrm{~N}$ squamous cell carcinoma.

The primary tumor, nodes, metastasis (TNM) classification of the American Joint Committee on Cancer/Union for International Cancer Control is a widely accepted staging system. The tumor stage impacts treatment decisions, predicts prognosis, and helps determine the patient's eligibility for various clinical trials.

Received September 10, 2015; accepted after revision January 18, 2016.

From the Departments of Radiation Oncology (B.K., U.P.) and Radiology (Y.A.), University of Washington, Seattle, Washington; Department of Radiology and Imaging Sciences (P.A.H.), Emory University, Atlanta, Georgia; and Department of Radiology and Imaging Sciences (Y.A.), University of Utah Health Care, Salt Lake City, Utah.

Please address correspondence to Yoshimi Anzai, MD, MPH, Department of Radiology and Imaging Sciences, University of Utah, 30 North 1900 East, 1A071, Salt Lake City, UT 84132; e-mail: Yoshimi.Anzai@hsc.utah.edu; @yoshimianzai

http://dx.doi.org/10.3174/ajnr.A4742
An explicit statement of the American Joint Committee on Cancer TNM classification in radiology reports has not been the standard practice to date. ${ }^{3,5}$ The value of incorporating staging information into radiology reports on treatment decisions or, ultimately, outcomes has not been previously studied or reported, to our knowledge.

Currently, there are no data regarding how often TNM staging information is routinely incorporated into radiology reports for patients with $\mathrm{H} \& \mathrm{~N}$ squamous cell carcinoma. We conducted a national survey to determine whether radiologists routinely report the stage of the primary tumor and metastatic nodes in their final imaging interpretation in patients with $\mathrm{H} \& \mathrm{~N}$ cancer. In addition, we explored the perceived barriers and values of incorporating TN staging among radiologists. Because cross-sectional imaging was emphasized, and not PET/CT, questions regarding distant metastases were not included.

The aim of this pilot study, therefore, was to assess the current practice of assigning a TN stage in the radiology report. This information may improve understanding in current practice and permit an open discussion among radiologists, oncologists, surgeons, administrators, and payers regarding how standardized 
Survey Questions

Question 1. Nature of practice:
a) Academic,
b) private
c) both

Question 2. Years of Experience
a) $<2$ years,
b) 2-5 years,
c) 5-8 years
d) $>8$ years

Question 3. Do you sub-specialize in Head and Neck Radiology?

a) Yes, b) No

Question 4. Do you routinely issue a radiology report using $\mathrm{TN}(\mathrm{M})$ staging?

a) Yes, b) No

Question 5. In your opinion, what are the barriers that might prevent radiologists from using the TN(M) staging system during reporting? (Check all that apply)
a) Afraid of inaccuracy
b) Time-consuming
c) Unable to remember the staging classification
d) No reimbursement
e) Not required
f) Other: (Space included for comments)

Question 6. If you are currently incorporating the TN(M) staging system, what are the reasons for this practice? (Check all that apply)
a) Believe in added values required from surgery or oncology colleagues
b) Educational value
c) Help the treatment decision
d) Not applicable
e) Other: (Space included for comments)

Question 7. How do you measure the size of primary H\&N tumor?
a) One dimension in the axial plane,
b) Bi-dimension in the axial plane,
c) Three dimensions including the cranio-caudal dimension

Question 8. How do you measure cervical lymph nodes?
a) Short axial dimension,
b) Longest axial dimension
c) Bi-dimension
d) Three dimensions including the cranio-caudal dimension

Question 9. How important do you think it is to incorporate TN(M) staging into a radiology report?
a) Very important,
b) Somewhat important,
d) Not very important, e) Not important
c) Neutral,

FIG 1. Survey questions.

radiology reports could potentially add value to the care of the patient with $\mathrm{H} \& \mathrm{~N}$ cancer. ${ }^{6}$

\section{MATERIALS AND METHODS Procedure}

The study was approved by University of Washington institutional review board. An electronic survey (Fig 1) was sent to 782 active members of the American Society of Head and Neck Radiology via their e-mail address in April 2014. Nonresponders were contacted with a reminder after 1 week. Data were collected anonymously by using Catalyst software (http://www.amd.com/ en-us/innovations/software-technologies/catalyst) to protect responders' privacy. Participants were not required to answer all questions and could choose to omit certain responses according to their level of comfort.
The survey asked the following questions: 1) the nature of practice (academic versus private); 2) years of experience; 3) whether the radiologist subspecialized in $\mathrm{H} \& \mathrm{~N}$ radiology, determined by self-identification; 4) their practice of routine assignment of $\mathrm{TN}$ staging in CT/MR reports; 5) the reasons for not explicitly reporting the stage in reports; or 6) the reasons for assigning the stage in reports; and 7) the importance of incorporating TN or TNM staging in a radiology report.

In addition, we asked the method of measuring the primary tumor and cervical lymph node size because these measures impact the assignment of $\mathrm{T}$ and $\mathrm{N}$ staging. The different methods of measuring can have varying prognosis and treatment modalities. ${ }^{4,7,8}$

Figure 1 shows the copy of survey.

\section{Instrument}

Questions were formulated with multiple-choice answers by using the Catalyst software. Seven questions were limited to only 1 choice, whereas 2 questions provided the possibility of choosing multiple answers. The 2 questions, 5) What are the reasons you do not explicitly report the stage in reports, and 6) What are the reasons you do assign the stage in reports, also included an additional "Other" answer with a comment box available for any responses not listed as an answer choice.

\section{Statistical Analysis}

Response statistics were given in the Catalyst software. Data were analyzed by using standard descriptive statistics. Further frequency tabulation and descriptive statistics were calculated by using Excel, Version 14.4.4 (Microsoft, Redmond, Washington). Confidence interval calculation was performed by using The Survey System (http://www.surveysystem.com/sscalc.htm).

\section{RESULTS}

\section{Participant Demographics}

A total of 229 responses were received within 2 weeks $(29.3 \%$ response rate). More than $62.8 \%$ (95\% CI, 57.5\%-68.0\%) of the responders were in an academic practice, $23.8 \%$ (95\% CI, $19.2 \%-28.4 \%)$ of responders were in private practice, and $13.5 \%$ (95\% CI, 9.8\%-17.2\%) reported being in combined private and academic practice; $63.3 \%$ had $>8$ years of experience, $12.7 \%$ of the responders had 5-8 years of experience, $17.0 \%$ had 2-5 years of experience, and $7.0 \%$ of responders had $<2$ 
Table 1: Survey responder demographics

\begin{tabular}{lcc}
\hline \multicolumn{1}{c}{ Variable } & No. of Responses & Frequency \\
\hline Nature of Practice & 229 & \\
Academic & 144 & $62.8 \%$ \\
Private & 54 & $23.8 \%$ \\
Both & 31 & $13.5 \%$ \\
Years of Experience & 229 & \\
$\quad<2$ years & 16 & $7.0 \%$ \\
2-5 years & 39 & $17.0 \%$ \\
5-8 years & 29 & $12.7 \%$ \\
>8 years & 145 & $63.3 \%$ \\
Subspecialty & 229 & \\
Head and neck radiology & 165 & $72.1 \%$ \\
Other subspecialization & 64 & $27.9 \%$ \\
$\quad$ of radiology & & \\
\hline
\end{tabular}

Table 2: Perceived barriers to incorporating staging in radiology reports $^{\mathrm{a}}$

\begin{tabular}{lcc}
\hline \multicolumn{1}{c}{ Answer } & $\begin{array}{c}\text { No. of Responses } \\
(\boldsymbol{n}=\mathbf{2 2 7})\end{array}$ & Frequency \\
\hline Afraid of inaccuracy & 134 & $59.0 \%$ \\
Unable to remember staging & 132 & $58.2 \%$ \\
$\quad$ classification & & \\
Time-consuming & 106 & $46.7 \%$ \\
Not required & 81 & $35.7 \%$ \\
Other & 74 & $32.6 \%$ \\
No reimbursement & 41 & $18.1 \%$ \\
\hline
\end{tabular}

a The sum of responses exceeds the total number of responses $(n=227)$ because participants were able to choose multiple answers for this particular question.

years of experience. Subspecialization in H\&N radiology was reported by $72.1 \%$ of respondents (Table 1 ).

\section{Reasons for Not Incorporating TN Staging in Routine Radiology Reports}

Of the total participants, $24.5 \%$ stated that they routinely assigned TN staging in radiology reports. Furthermore, 30.3\% (95\% CI, $24 \%-36.5 \%$ ) of those who subspecialize in $\mathrm{H} \& \mathrm{~N}$ radiology routinely perform TN staging. The 2 most frequent responses to potential reasons for not stating the $\mathrm{TN}$ stage in reports or barriers to such staging were fear of inaccuracies and being unable to remember the staging classification (Table 2). These responses may be due to the periodic changes of TNM classification ${ }^{9}$ because the system is reviewed every 7-10 years and there are changes in the staging system at various subsites. Approximately one-third thought that reporting staging was not required, and 18\% did not give the stage due to lack of reimbursement.

Of the $32.6 \%$ who replied "other," the answers were broadly categorized as follows: $40.5 \%$ responded that insufficient clinical data were provided to accurately report TN staging and that staging without considering clinical data was not advised. For example, vocal cord mobility is an important factor for staging laryngeal cancer and cannot always be accurately determined solely on imaging. ${ }^{10} 16.2 \%$ stated that referring physicians prefer to do it themselves to prevent conflicting readings and that referring physicians prefer not to have radiology TNM staging in the report. $13.5 \%$ responded that their imaging interpretation including all necessary staging information, just not in the final TN staging. Staging being done with referring physicians at a Tumor Board setting was reported in $10.8 \%$. Not interpreting PET scans that are essential for staging was reported in $8.1 \%$. Finally, 5.4\% reported
Table 3: Reasons for assigning $\mathrm{TN}$ staging in radiology reports ${ }^{\mathrm{a}}$

\begin{tabular}{lcc}
\hline \multicolumn{1}{c}{ Answer } & $\begin{array}{c}\text { No. of Responses } \\
(\boldsymbol{n}=115)\end{array}$ & Frequency \\
\hline $\begin{array}{l}\text { Believe in added value required } \\
\text { from surgery or oncology }\end{array}$ & 60 & $52.2 \%$ \\
$\quad$ colleagues & & \\
Help the treatment decision & 50 & $43.5 \%$ \\
Not applicable & 40 & $34.8 \%$ \\
Educational value & 38 & $33.0 \%$ \\
Other & 17 & $14.8 \%$ \\
\hline
\end{tabular}

a The sum of responses exceeds the total number of responses $(n=115)$ because participants were able to choose multiple answers for this particular question.

that the referring clinician may not fully understand the complexity of staging based solely on imaging.

\section{Reasons for Incorporating TN Staging in Routine Radiology Reports}

Responders who routinely assign TN staging indicated that the reasons for such practice are the following; 1) They believe it adds value to the report, 2) it helps determine treatment decisions, and 3 ) it is of educational value (Table 3). "Not applicable" was available for those who did not routinely assign TN staging. The number of people who answered this question $(n=115)$ exceeded the number responding that they routinely assign TN staging. We speculate that some selected the reasons for incorporating TN staging on the basis of their expertise, despite not routinely assigning TN staging in their reports. Other responses included the following: 1) to speak same language as head and neck surgery colleagues, 2) marketing, 3) faster presentation at the Tumor Board, and 5) the pathology department follows the College of American Pathologists protocol to assign pTNM staging, as such radiologists should assign rTNM staging.

\section{Measurements of Primary Tumor}

The survey also asked how radiologists measure the size of a primary $\mathrm{H} \& \mathrm{~N}$ tumor, which is an important determinant of TN staging. Measuring a primary tumor in 3D was reported in $76.9 \%$ (95\% CI, $72 . \%-81.5 \%), 17.3 \%$ (95\% CI, $13.2 \%-21.4 \%$ ) of the responders measure a primary tumor in $2 \mathrm{D}$, and $5.8 \%(95 \% \mathrm{CI}$, $3 . \%-8.1 \%$ ) of participants measure a primary tumor in $1 \mathrm{D}$ (ie, in the axial plane; Figs 2 and 3).

\section{Measurements of Cervical Lymph Node Size}

Measurements of cervical lymph node size were quite variable. The survey showed $37.3 \%$ (95\% CI, 32\%-42.6\%) of respondents measured lymph nodes in the longest axial dimension, $17.3 \%$ (95\% CI, $13.2 \%-21.4 \%$ ) reported that they measure along the short axial dimension, $28.4 \%$ (95\% CI, 23.5\%-33.3\%) of responders measure cervical lymph nodes in 2 and $16.9 \%$ (95\% CI, 12.8\%-21\%) reponders measured in $3 \mathrm{D}$, including the craniocaudal dimension.

\section{Perceived Importance of Incorporating TN Staging into Routine Practice}

Approximately half (48.9\%) of the responders answered that assigning TN staging was important, and 34.9\% were neutral in their views. Only $16.2 \%$ thought it was not important (Table 4). However, among the respondents who indicated that TN staging was important, $53.6 \%$ were not incorporating the stage into their routine dictations. 

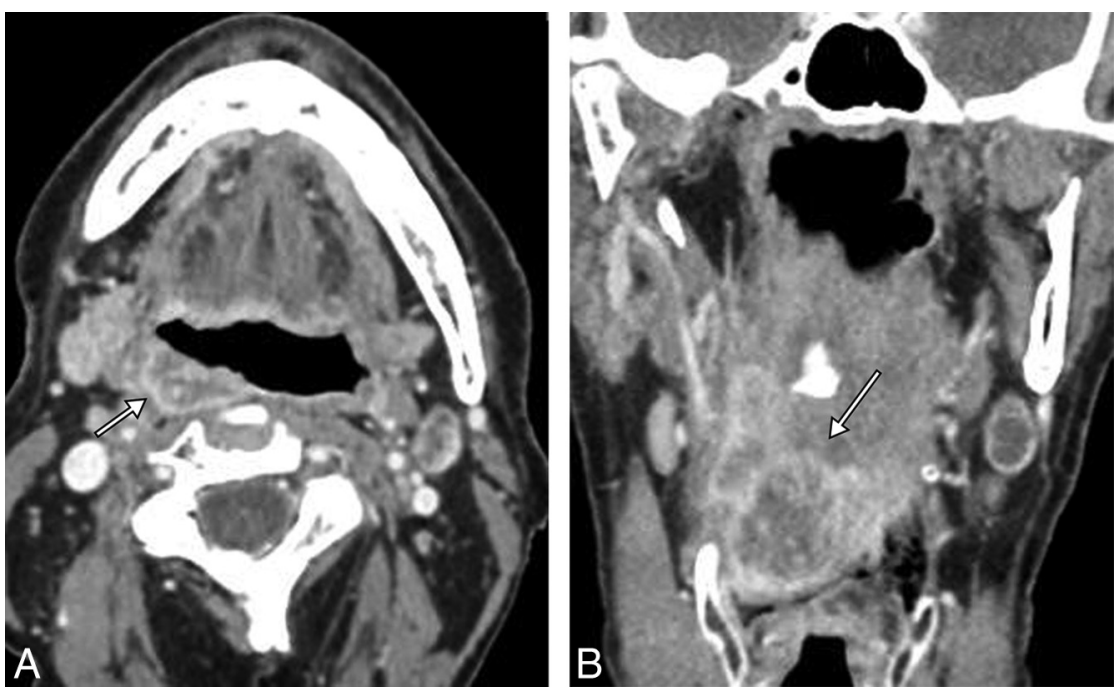

FIG 2. Contrast-enhanced axial $(A)$ and coronal $(B) C T$ images of a patient with posterior pharyngeal wall cancer. The transaxial dimension is $2.5 \mathrm{~cm}$ (short arrow), though the craniocaudal dimension exceeds $4 \mathrm{~cm}$ (long arrow). Therefore, based on size alone, the stage is T3. Addition of concurrent chemotherapy to radiation therapy would be appropriate in a T3, but not a T2 lesion. Additional factors that would upstage this tumor, as described in American Joint Committee on Cancer, 7th edition (https://cancerstaging.org/Pages/default.aspx) are extension to the larynx, involvement of extrinsic tongue muscles, medial pterygoid muscle involvement, or hard palate or mandible invasion. These are all imaging-based characteristics.
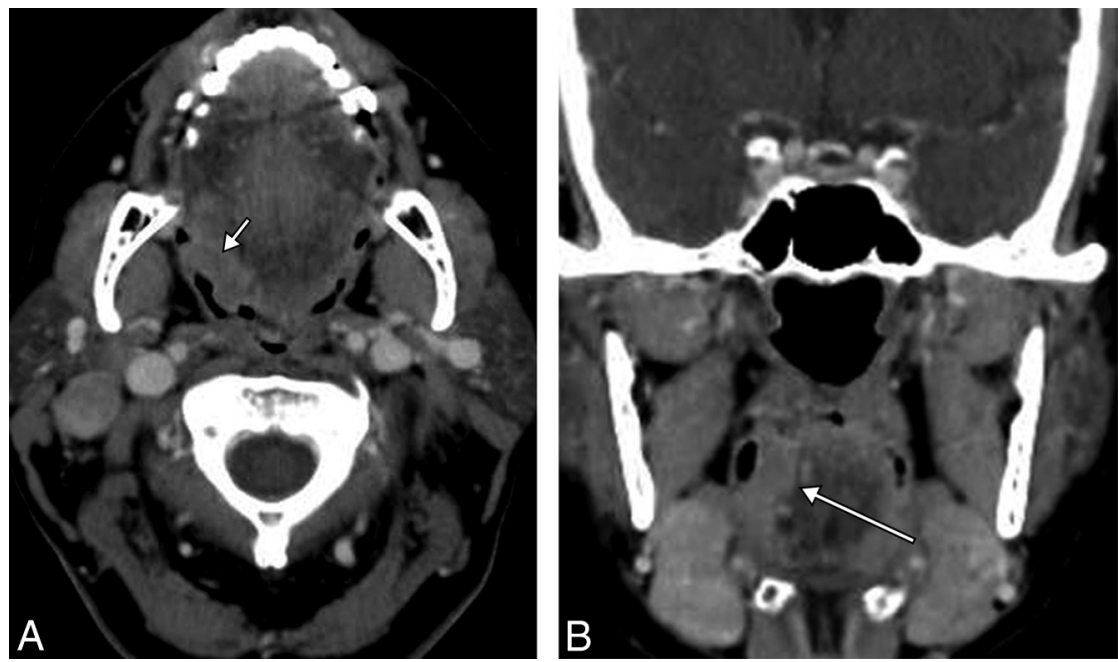

FIG 3. Contrast-enhanced axial $(A)$ and coronal $(B) C T$ images of a patient with squamous cell carcinoma of the right base of the tongue. Although the transaxial dimension is $1.8 \mathrm{~cm}$ (short arrow), the craniocaudal dimension is $3.5 \mathrm{~cm}$, technically at least a T2 lesion, if only the size is considered. These measurements can only be accurately acquired on imaging. Note that the largest craniocaudal dimension (long arrow) is submucosal, and on the basis of physical examination alone, this tumor could be grossly understaged.

\section{DISCUSSION}

We conducted this survey to understand the current practice among radiologists primarily in the United States regarding the use of the TNM staging, and more specifically the TN characteristics, in their routine reports. Our goal is to open a discussion among radiologists, referring physicians, administrators, and payers on how radiology reports could provide relevant staging information in an unambiguous fashion. ${ }^{11}$

To our knowledge, this is the first reported national survey regarding imaging-based staging for practicing neuroradiologists and $H \& N$ radiologists. We found that approximately half of the responding radiologists viewed it as important to incorporate $\mathrm{TN}$ staging in reports, though only $24.5 \%$ of radiologists routinely assign a stage in these reports.

One of the more common factors preventing radiologists from using $\mathrm{TN}$ staging appeared to be a lack of sufficient clinical data available to the radiologist. TN staging based solely on imaging findings is thus perceived to be inaccurate. Some radiologists thought that with incomplete history and clinical data, it was outside their scope of practice to routinely report formal TN staging. They also replied that applying TN staging on the basis of imaging alone can lead to incomplete data and may potentially carry medical-legal ramifications. For practices with robust electronic medical records allowing readily available clinical information, this concern may not be a major barrier. One subsite where staging might be limited by not knowing the clinical examination results is staging of laryngeal cancer, ${ }^{10}$ where vocal cord mobility would change the T stage. Another subsite where lack of clinical information may limit accurate imagingbased staging is oral cavity cancer, where mucosal extent is clearly assessed by the physical examination. Cross-sectional imaging does not demonstrate the extent of the superficial mucosal portion of the oral cavity lesion. If this information is not available in the electronic medical records, the interpretation could include all other imaging findings needed for staging.

Some responders thought that radiologists must provide surgeons and treating physicians with the relevant staging information, but it is treating physicians' responsibility to merge the clinical and radiology information to stage the patient. No studies to date have addressed how often radiology reports include all relevant staging information for treating physicians to determine accurate staging. A structured reporting system incorporating the American Joint Committee on Cancer staging system may help provide complete information necessary for staging each head and neck subsite. To mitigate the perceived limitations related to lack of clinical information, the radiology report could state, "Stage based on imaging alone is T2 N1," for example. Another method of reporting could be similar to the pathology report, by using a lowercase $r$, for example rT2 $\mathrm{N} 1$, similar to $\mathrm{CT} 2 \mathrm{~N} 1$, which represents staging based on clinical examination.

In addition, an important goal of cross-sectional imaging such 


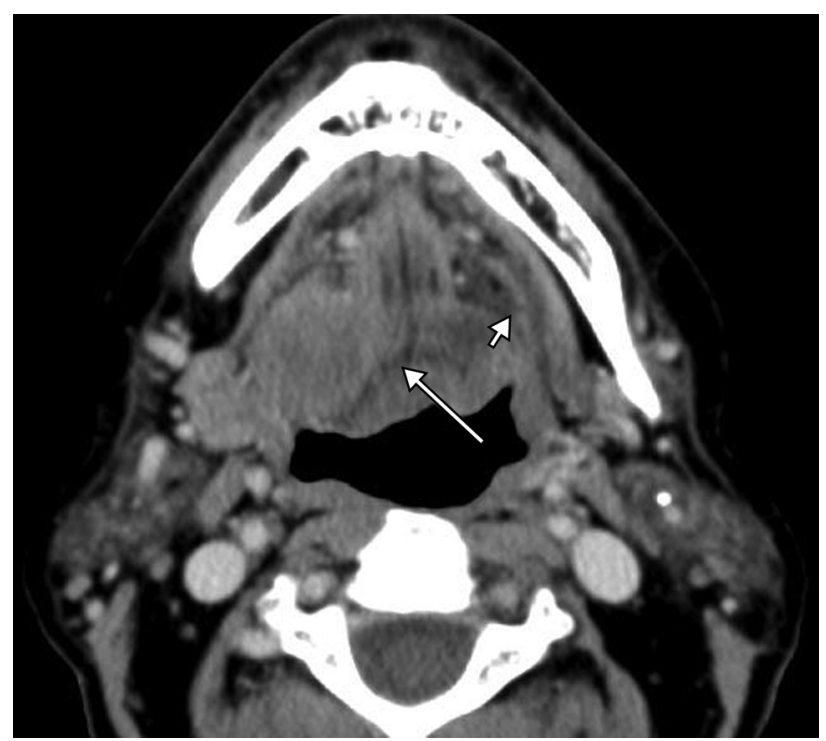

FIG 4. Axial contrast-enhanced $C T$ at the floor of mouth level. Clinically, this tumor was staged as T2 right oral cavity squamous cell carcinoma because there was mucosal ulceration and a $>2-\mathrm{cm}$ palpable mass. However, there is invasion of the lateral and posterior genioglossus muscle (long arrow). Note a normal hyoglossus muscle on the left (short arrow); tumor has completely replaced right hyoglossus muscle. This is upstaged to T4a on the basis of CT findings, changing the prognosis and treatment.

Table 4: Perceived importance of incorporating TN staging in radiology reports

\begin{tabular}{lcc}
\hline \multicolumn{1}{c}{ Answer } & No. of Responses $(\boldsymbol{n}=\mathbf{2 2 9})$ & Frequency \\
\hline Very important & 44 & $19.2 \%$ \\
Somewhat important & 68 & $29.7 \%$ \\
Neutral & 80 & $34.9 \%$ \\
Not very important & 18 & $7.9 \%$ \\
Not important & 19 & $8.3 \%$ \\
\hline
\end{tabular}

as CT or MR imaging is to address the deeper extension of tumor (Fig 4), not to measure the superficial mucosal extent of a tumor. A superficial tongue lesion with no deep invasion may not be visible on CT or MR imaging and therefore would be potentially understaged on the basis of imaging alone. Contrary to other body sites, such as lung cancer in which staging is based solely on imaging findings, the staging of $\mathrm{H} \& \mathrm{~N}$ cancer sometimes requires knowledge of the clinical findings. In these settings, the dictation can be as thorough as possible so that Tumor Board members can assign a stage. These examples support the abovementioned concerns of radiologists, and such limitations should be acknowledged. A multidisciplinary Tumor Board setting is an ideal situation to discuss the clinical and imaging findings and assign a single TN stage, so that proper treatment can be planned.

This survey revealed that $76.9 \%$ of the responders measure a primary $\mathrm{H} \& \mathrm{~N}$ tumor in $3 \mathrm{D}$. It is critically important because the staging depends on the largest dimension of a tumor, which is not necessarily the largest transaxial dimension (Fig 2). For example, a $1.8-\mathrm{cm}$ tonsil cancer in the transaxial dimension may extend more than $2 \mathrm{~cm}$ in the craniocaudal dimension, which should be staged at least T2 based on size, and not T1 (Fig 3). Even though response evaluation criteria in solid tumors require only $1 \mathrm{D}$ measurement to address the therapeutic effect of investigational drugs in clinical trials, 3D measurement of $\mathrm{H} \& \mathrm{~N}$ cancer more accurately reflects real tumor burden in an individual patient. ${ }^{12,13}$

The measurement of cervical lymph nodes was more variable than that for primary tumors among the survey responders. It has been reported that the short axial diameter of lymph nodes is the most accurate indicator of metastatic versus normal or reactive nodes. ${ }^{8,14}$ The largest diameter of the lymph node also affects the nodal staging, which is not necessarily the largest transaxial diameter of the lymph nodes. With the advancement of CT technology and isometric voxel size, coronal or sagittal reformatted images are routinely obtained in most current practices. ${ }^{15}$ Thus, measuring the largest dimension of a lymph node is essential for accurate nodal staging.

TN staging in radiology reports is critical in providing essential information for treatment planning and prognosis of various cancers. ${ }^{16,17}$ It allows precise preoperative extent of tumor, which enhances levels of objectivity of clinical staging based on physical examination. ${ }^{18}$ The Quality and Productivity: Proposed Case Study performed by the National Institute for Health and Care Excellence in the United Kingdom demonstrated that TNM classification not explicitly stated but rather implied in free text format led to delays in treatment or incorrect decisions regarding treatment. ${ }^{19}$ This study showed that structured radiology reports that include explicit TNM staging for cervical, endometrial, ovarian, prostate, and rectal cancer improved prognostic accuracy and reduced cost on the basis of reduced time seeking clarification, with approximate saving of $£ 2900$ (US $\$ 4,150$ ) per 100,000 population (www.evidence.nhs.uk/qualityandproductivity). ${ }^{19}$

One of the limitations of the current study is that it was sent to only members of the American Society of Head and Neck Radiology. These results may not apply to a broader and less specialized group of radiologists. The argument can be made that all oncologic studies should be interpreted and reported by subspecialists because treatment regimens vary, on the basis almost entirely of the stage. The importance of providing accurate staging, especially in the $\mathrm{H} \& \mathrm{~N}$, cannot be overemphasized. A future study might include the assessment of the clinical practice of incorporating FDG-PET for staging $\mathrm{H} \& \mathrm{~N}$ cancer $^{20,21}$ and reporting of TNM staging of H\&N cancer on FDG-PET.

\section{CONCLUSIONS}

Staging H\&N cancer based on imaging presents unique challenges, but also opportunities. Although some H\&N cancers may not be accurately staged with imaging, staging for most $\mathrm{H} \& \mathrm{~N}$ cancer is determined by the size and local extent, best noted on cross-sectional imaging, often in conjunction with PET. Future study is needed to determine whether assigning an imaging-based stage could improve treatment decisions for patients with $\mathrm{H} \& \mathrm{~N}$ cancer or impact referring physicians' and patients' satisfaction.

\section{REFERENCES}

1. Curtin HD, Ishwaran H, Mancuso AA, et al. Comparison of CT and MR imaging in staging of neck metastases. Radiology 1998;207: 123-30 CrossRef Medline

2. Hudgins PA, Kingdom TT, Weissler MC, et al. Selective neck dissection: CT and MR imaging findings. AJNR Am J Neuroradiol 2005;26:1174-77 Medline 
3. Yousem DM, Gad K, Tufano RP. Resectability issues with head and neck cancer. AJNR Am J Neuroradiol 2006;27:2024-36 Medline

4. Mukherji SK, Mancuso AA, Mendenhall W, et al. Can pretreatment CT predict local control of $\mathrm{T} 2$ glottic carcinomas treated with radiation therapy alone? AJNR Am J Neuroradiol 1995;16:655-62 Medline

5. Patel SG, Shah JP. TNM staging of cancers of the head and neck: striving for uniformity among diversity. CA Cancer J Clin 2005;55: 242-58; quiz 261-62, 64 Medline

6. Knechtges PM, Carlos RC. The evolving role of radiologists within the health care system. J Am Coll Radiol 2007;4:626-35 CrossRef Medline

7. Mancuso AA, Harnsberger HR, Muraki AS, et al. Computed tomography of cervical and retropharyngeal lymph nodes: normal anatomy, variants of normal, and applications in staging head and neck cancer, Part II: pathology. Radiology 1983;148:715-23 CrossRef Medline

8. van den Brekel MW, Castelijns JA, Snow GB. The size of lymph nodes in the neck on sonograms as a radiologic criterion for metastasis: how reliable is it? AJNR Am J Neuroradiol 1998;19:695-700 Medline

9. Takes RP, Rinaldo A, Silver CE, et al.. Future of the TNM classification and staging system in head and neck cancer. Head Neck 2010; 32:1693-711 CrossRef Medline

10. Gilbert K, Dalley RW, Maronian N, et al. Staging of laryngeal cancer using 64-channel multidetector row CT: comparison of standard neck CT with dedicated breath-maneuver laryngeal CT. AJNR AmJ Neuroradiol 2010;31:251-56 CrossRef Medline

11. Glastonbury CM, Bhosale PR, Choyke PL, et al. Do radiologists have stage fright? Tumor staging and how we can add value to the care of patients with cancer. Radiology 2016;278:11-12 CrossRef Medline

12. Street E, Hadjiiski L, Sahiner B, et al. Automated volume analysis of head and neck lesions on CT scans using 3D level set segmentation. Med Phys 2007;34:4399-408 CrossRef Medline

13. Hadjiiski L, Mukherji SK, Gujar SK, et al. Treatment response assessment of head and neck cancers on CT using computerized volume analysis. AJNR Am J Neuroradiol 2010;31:1744-51 CrossRef Medline

14. van den Brekel MW, Stel HV, Castelijns JA, et al. Cervical lymph node metastasis: assessment of radiologic criteria. Radiology 1990; 177:379-84 CrossRef Medline

15. Kato H, Kanematsu M, Watanabe $\mathrm{H}$, et al. Metastatic retropharyngeal lymph nodes: comparison of $\mathrm{CT}$ and MR imaging for diagnostic accuracy. Eur J Radiol 2014;83:1157-62 CrossRef Medline

16. UyBico SJ, Wu CC, Suh RD, et al. Lung cancer staging essentials: the new TNM staging system and potential imaging pitfalls. Radiographics 2010;30:1163-81 CrossRef Medline

17. Lee SC, Jain PA, Jethwa SC, et al. Radiologist's role in breast cancer staging: providing key information for clinicians. Radiographics 2014;34:330-42 CrossRef Medline

18. Schwartz LH, Panicek DM, Berk AR, et al. Improving communication of diagnostic radiology findings through structured reporting. Radiology 2011;260:174-81 CrossRef Medline

19. Addenbrooke's Hospital, Cambridge University Hospitals NHS Foundation Trust. Template reports for radiology cancer staging: improving information to guide treatment decisions. 2014. www. evidence.nhs.uk/qualityandproductivity. Accessed January 4, 2016

20. Roh JL, Park JP, Kim JS, et al. 18F fluorodeoxyglucose PET/CT in head and neck squamous cell carcinoma with negative neck palpation findings: a prospective study. Radiology 2014;271:153-61 CrossRef Medline

21. Mukherji SK, Bradford CR. Controversies: is there a role for positron-emission tomographic CT in the initial staging of head and neck squamous cell carcinoma? AJNR Am J Neuroradiol 2006;27: 243-45 Medline 